\title{
TWO INTEGRALS OF RAMANUJAN
}

\author{
RICHARD ASKEY 1
}

ABstract. Two integrals of Ramanujan are evaluated.

In the pages of identities of Ramanujan that G. Andrews found in 1976 [2], there is one page of integrals related to the normal integral. Derivations of Ramanujan's identities are given below. For ease in printing, set $q=e^{-2 k^{2}}$ and

$$
(a ; q)_{\infty}=\prod_{n=0}^{\infty}\left(1-a q^{n}\right)
$$

Ramanujan stated the following identities:

$$
\begin{gathered}
\int_{-\infty}^{\infty} e^{-x^{2}+2 m x}\left(-a e^{2 k x} q ; q\right)_{\infty}\left(b e^{-2 k x} q ; q\right)_{\infty} d x \\
=\frac{\sqrt{\pi}(a b q ; q)_{\infty} e^{m^{2}}}{\left(a q^{1 / 2} e^{2 m k} ; q\right)_{\infty}\left(b q^{1 / 2} e^{-2 m k} ; q\right)_{\infty}}, \quad q=e^{-2 k^{2}}, \\
\int_{-\infty}^{\infty} \frac{e^{-x^{2}+2 m x} d x}{\left(a q^{1 / 2} e^{2 i k x} ; q\right)_{\infty}\left(b q^{1 / 2} e^{-2 i k x} ; q\right)_{\infty}} \\
=\sqrt{\pi} e^{m^{2}} \frac{\left(-a q e^{2 i m k} ; q\right)_{\infty}\left(-b q e^{-2 i m k} ; q\right)_{\infty}}{(a b q ; q)_{\infty}}, \quad q=e^{-2 k^{2}} .
\end{gathered}
$$

To obtain these results we will use the $q$-binomial theorem

$$
\sum_{n=0}^{\infty} \frac{(a ; q)_{n}}{(q ; q)_{n}} x^{n}=\frac{(a x ; q)_{\infty}}{(x ; q)_{\infty}}, \quad|q|<1, \quad|x|<1,
$$

and a limiting case of it

$$
\sum_{n=0}^{\infty} \frac{(-1)^{n} q^{\left(n^{2}-n\right) / 2} x^{n}}{(q ; q)_{n}}=(x ; q)_{\infty}, \quad|q|<1,
$$

where

$$
(a ; q)_{n}=(a ; q)_{\infty} /\left(a q^{n} ; q\right)_{\infty}
$$

See [1, Theorem 2.1], [3] or [4, p. 66] for proofs.

The parameter $m$ in (2) and (3) can be removed by translation and redefinition of $a$ and $b, 80$ we assume $m=0$. To prove (2) use (5) on each of the infinite products

Received by the editors July 31, 1981.

1980 Mathematics Subject Classification. Primary 33A15.

Key words and phrases. Ramanujan, integrals.

${ }^{1}$ Supported in part by NSF grant MCS-8101568. 
to obtain

$$
\begin{aligned}
\int_{-\infty}^{\infty} e^{-x^{2}} & \left(-a e^{2 k x} q ; q\right)_{\infty}\left(-b e^{-2 k x} q ; q\right)_{\infty} d x \\
& =\sum_{n=0}^{\infty} \sum_{m=0}^{\infty} \frac{q^{\left(n^{2}+n\right) / 2} a^{n}}{(q ; q)_{n}} \frac{q^{\left(m^{2}+m\right) / 2} b^{m}}{(q ; q)_{m}} \int_{-\infty}^{\infty} e^{-x^{2}+2 k(n-m) x} d x \\
& =\sqrt{\pi} \sum_{n, m=0}^{\infty} \frac{q^{\left(n^{2}+n\right) / 2+\left(m^{2}+m\right) / 2} a^{n} b^{m}}{(q ; q)_{n}(q ; q)_{m}} e^{k^{2}(n-m)^{2}} \\
& =\sqrt{\pi} \sum_{n, m=0}^{\infty} \frac{q^{n / 2+m / 2+n m} a^{n} b^{m}}{(q ; q)_{n}(q ; q)_{m}} \\
& =\sqrt{\pi} \sum_{n=0}^{\infty} q^{n / 2} \frac{a^{n}}{(q ; q)_{n}} \frac{1}{\left(b q^{n+1 / 2} ; q\right)_{\infty}} \quad(\text { by }(4) \text { with } a=0) \\
& =\frac{\sqrt{\pi}}{\left(b q^{1 / 2} ; q\right)_{\infty}} \sum_{n=0}^{\infty} \frac{\left(b q^{1 / 2} ; q\right)_{n}\left(a q^{1 / 2}\right)^{n}}{(q ; q)_{n}} \\
& =\frac{\sqrt{\pi}(a b q ; q)_{\infty}}{\left(a q^{1 / 2} ; q\right)_{\infty}\left(b q^{1 / 2} ; q\right)_{\infty}} \quad(\text { by }(4)) .
\end{aligned}
$$

To obtain (3) use (4) with $a=0$ as follows.

$$
\begin{aligned}
\int_{-\infty}^{\infty} & \frac{e^{-x^{2}} d x}{\left(a q^{1 / 2} e^{2 i k x} ; q\right)_{\infty}\left(b q^{1 / 2} e^{-2 i k x} ; q\right)_{\infty}} \\
& =\sum_{n, m=0}^{\infty} \frac{a^{n} q^{n / 2} b^{m} q^{m / 2}}{(q ; q)_{n}(q ; q)_{m}} \int_{-\infty}^{\infty} e^{-x^{2}+2 i k(n-m) x} d x \\
& =\sqrt{\pi} \sum_{n, m=0}^{\infty} \frac{a^{n} q^{n / 2} b^{m} q^{m / 2}}{(q ; q)_{n}(q ; q)_{m}} e^{-k^{2}(n-m)^{2}} \\
& =\sqrt{\pi} \sum_{m, n=0}^{\infty} \frac{a^{n} q^{n / 2} b^{m} q^{m / 2} q^{n^{2} / 2+m^{2} / 2-m n}}{(q ; q)_{n}(q ; q)_{m}} \\
& =\sqrt{\pi} \sum_{n=0}^{\infty} \frac{a^{n} q^{\left(n^{2}+n\right) / 2}}{(q ; q)_{n}} \sum_{m=0}^{\infty} \frac{\left(b q^{1-n}\right)^{m} q^{\left(m^{2}-m\right) / 2}}{(q ; q)_{m}} \\
& =\sqrt{\pi} \sum_{n=0}^{\infty} \frac{a^{n} q^{\left(n^{2}+n\right) / 2}}{(q ; q)_{n}}\left(-b q^{1-n} ; q\right)_{\infty} \quad(b y(5)) \\
& =\sqrt{\pi}(-b q ; q)_{\infty} \sum_{n=0}^{\infty} \frac{a^{n} q^{\left(n^{2}+n\right) / 2}\left(-b q^{1-n} ; q\right)_{n}}{(q ; q)_{n}} \\
& =\sqrt{\pi}(-b q ; q)_{\infty} \sum_{n=0}^{\infty}(a b q)^{n} \frac{(-1 / b ; q)_{n}}{(q ; q)_{n}} \\
& =\sqrt{\pi} \frac{(-b q ; q)_{\infty}(-a q ; q)_{\infty}}{(a b q ; q)_{\infty}}(b y(4)) .
\end{aligned}
$$

Hardy remarked [5, p. xxv], "There is always more in one of Ramanujan's formulae than meets the eye, as anyone who gets to work to verify those which look the easiest will soon discover. In some the interest lies very deep, in others 
comparatively near the surface; but there is not one which is not curious and entertaining." I cannot tell yet what the real interest is in (2) and (3). However I have only known them for five years, and it took me over ten years to understand another integral of Ramanujan [3].

\section{REFERENCES}

1. G. E. Andrews, The theory of partitions, Addison-Wesley, Reading, Mass., 1976.

2. __ An introduction to Ramanujan's "lost" notebook, Amer. Math. Monthly 86 (1979), 89-108.

3. R. Askey, Ramanujan's extensions of the gamma and beta functions, Amer. Math. Monthly 87 (1980), 346-359.

4. W. N. Bailey, Generalized hypergeometric series, Cambridge Univ. Press, 1935; reprinted, Hafner, New York, 1972.

5. S. Ramanujan, Collected papers, edited by G. H. Hardy, P. V. Seshu Aiyar and B. M. Wilson, Cambridge Univ. Press, 1927; reprinted, Chelsea, New York, 1959.

Department of Mathematics, VAN Vleck hall, UNIVERsity of WisconsinMADISON, MADISON, WISCONSIN 53706 